\title{
Paraesophageal hiatal hernia. Open vs. laparoscopic surgery
}

\author{
M. Díez Tabernilla, J. Ruiz-Tovar, R. Grajal Marino, P. Calero García, J. D. Pina Hernández, A. Chames \\ Vaisman, V. Morales Castiñeiras, A. Sanjuanbenito Dehesa, E. Martínez Molina and V. Fresneda Moreno \\ Service of General Surgery and Digestive Diseases. University Hospital Ramón y Cajal. Madrid, Spain
}

\begin{abstract}
Background: paraesophageal hiatal hernia represents 5-10\% of hiatal hernias. Its importance is based on the severe complications it may have, including gastric volvulus, and surgical treatment is recommended when a diagnosis is established.

Material and methods: a retrospective study of all patients who underwent surgery for paresophageal hernia between 1985 and 2007.

Results: we studied 90 cases, 68 females and 22 males with a median age of 67.6 years (37-96). Forty-five patients reported pyrosis, 34 epigastric postprandial pain, and 15 dysphagia; eight patients were diagnosed with gastric volvulus. Eighty-one patients underwent elective surgery and 9 emergency surgery. Forty-seven cases underwent an open procedure and 43 a laparoscopic one; 5 (11.6\%) of them required conversion. The techniques performed were D'Or fundoplication in 35 cases, Nissen in 35, Toupet in 14, simple hiatal closure in 2, Narbona in 1, and Lortat-Jakob in 1; in 10 patients a mesh was placed. The complication rate for open procedure was 10.6 and $9.5 \%$ for the laparoscopic one ( $p>0.05)$. Median hospital stay was 9.1 days for the open procedure and 3.4 for the laparoscopic one $(p<0.05)$. As follow-up, we analyzed 84 patients. After a median follow-up of 12 years (1-19), 15 patients were still symptomatic (17.8\%), with recurrence in 8 cases ( 5 required reoperation). The satisfaction rate was $95.5 \%$

Conclusion: equivalent results were observed after laparoscopic and open surgery and a significant shorter hospital stay in the laparoscopic one. Therefore, we think that laparoscopic surgery should be considered as the election procedure for paraesophageal hiatal hernia.
\end{abstract}

Key words: Paraesophageal hernia. Hiatal hernia. Laparoscopy. Hernia repair.

Received: 05-02-09.

Accepted: 12-06-09.

Correspondence: María Díez Tabernilla. C/ Padre Francisco Palau y Quer, 7,4ํㅡ. 28046 Madrid. e-mail: mariadieztabernilla@gmail.com
Díez Tabernilla M, Ruiz-Tovar J, Grajal Marino R, Calero García P, Pina Hernández JD, Chames Vaisman A, Morales Castiñeiras V, Sanjuanbenito Dehesa A, Martínez Molina E, Fresneda Moreno V. Paraesophageal hiatal hernia. Open us. laparoscopic surgery. Rev Esp Enferm Dig 2009; 101: 706711.

\section{INTRODUCTION}

Paraesophageal hiatal hernia is an uncommon condition which represents 5-10\% of all hiatal hernias. Its importance is based on the severe complications it may have, such as obstruction, gastric perforation or gastric mucosal bleeding. This is the reason why treatment is recommended for all cases (1-3), even if some authors question this attitude and advocate for an expecting attitude in selected cases $(4,5)$. The open procedure (OP) shows high complication rates and needs a long recovering time $(1,6)$. In most recent series, the laparoscopic procedure (LP) has proven to be feasible and safe, and entails shorter hospital stays and lower complication rates, even though some authors suggest higher recurrence rates (7).

\section{MATERIAL AND METHODS}

We retrospectively reviewed all patients who underwent surgery for paraesophageal hernia between 1985 and 2007. Data were collected on age, sex, clinical signs and symptoms, diagnostic studies, surgical technique, postoperative complications, and hospital stay.

To evaluate long-term results a survey was performed between January and April 2008, inquiring on recurrence and patient satisfaction. Deceased patients and those lost to follow-up were excluded. 
A statistical analysis was performed using the Windows SPSS 12.0 program. Quantitative variables were defined by mean (median in no-Gaussian variables) and interval values; for discrete variables the number of cases and percentages were used. A comparison of discrete variables was performed using Student's t-method (Mann-Whitney for non-normal variables). Statistical significance was considered for $\mathrm{p}<0.05$

\section{RESULTS}

Ninety patients were included, 22 males (24.4\%) and 68 females $(75.6 \%)$, with a median age of 67.6 years (37-96). Surgical treatment was indicated in all cases referred to our general surgery clinic, excluding those whose comorbidity contraindicated the surgical procedure. Clinical manifestations are showed in table I. Predominant symptoms were pyrosis $(50 \%)$, epigastric postprandial pain $(37.5 \%)$, gastric volvulus $(8.9 \%)$, and dysphagia (5.6\%). Diagnostic studies are summarized in table II. Chest X-rays were performed for $83 \%$ of cases, these being important to establish diagnostic suspicion, as well as for cases of gastric volvulus. Upper endoscopy and gastroduodenal radiology with barium were the studies with the highest diagnostic utility, and confirmed the diagnosis in $91 \%$ of cases. $\mathrm{pH}-$ metry provided information about the presence of gastroesophageal reflux (GER), and manometry informed about the esophageal dynamic function.

Eighty-one patients underwent elective surgery $(90 \%)$ and 9 emergency surgery (10\%) (8 for gastric volvulus and one for massive gastrointestinal bleeding). Emergency surgery was always an OP. Surgical procedures performed are shown in table III. Forty-seven cases un-

Table I. Clinical manifestations

\begin{tabular}{lc}
\hline & Number of cases \\
\hline Pyrosis & $45(50 \%)$ \\
Epigastric pain & $34(37.8 \%)$ \\
Gastric volvulus & $8(8.9 \%)$ \\
Dysphagia & $5(5.6 \%)$ \\
Dyspnea & $5(5.6 \%)$ \\
UGIB & $5(5.6 \%)$ \\
Vomiting & $4(4.4 \%)$ \\
Food regurgitation & $3(3.3 \%)$ \\
\hline
\end{tabular}

*UGIB: upper gastro-intestinal bleeding.

Table II. Diagnostic studies

\begin{tabular}{lc}
\hline & Number of cases \\
\hline UGIE* $^{*}$ & $40(44.4 \%)$ \\
pH-metry & $16(17.8 \%)$ \\
EGD** & $63(70 \%)$ \\
Manometry & $19(21 \%)$ \\
Chest X-rays & $75(83.3 \%)$ \\
\hline
\end{tabular}

*UGIE: upper gastro-intestinal endoscopy; **EGD: esophagogastroduodenography with barium contrast.
Table III. Surgical technique

\begin{tabular}{lcc}
\hline Surgical technique & $O P$ & $L P$ \\
\hline D'Or & 35 & 0 \\
Nissen & 5 & 30 \\
Toupet & 3 & 13 \\
Simply pillars closure & 2 & 0 \\
Narbona & 1 & 0 \\
Lortat-Jakob & 1 & 0 \\
\hline Mesh placement & 2 & 8 \\
\hline
\end{tabular}

derwent an OP (52.2\%): surgical techniques chosen included D'Or fundoplication in 35 cases $(74.5 \%)$, Nissen in 8 cases $(17 \%)$, simple suture in $2(4.3 \%)$, Narbona in 1 case $(2.1 \%)$, and Lortat-Jakob in 1 case $(2.1 \%)$. Fortythree patients underwent LP (47.8) and 5 (11.6\%) of them required conversion. The surgical procedure was Nissen's for 27 cases $(62.8 \%)$ and Toupet's for 16 cases $(37.2 \%)$. A prosthetic mesh was placed in 8 cases during OP $(18.6 \%)$ and in 2 cases during LP $(4.3 \%)$.

Postoperative complications emerged in 5 cases $(10.6 \%)$ for OP and in 4 cases $(9.5 \%)$ for LP ( $p>0.05)$ (Table IV). Median hospital stay was 9 days for OP and 3.4 for LP ( $<<0.05)$. During follow-up we analyzed all other 84 patients $(93.3 \%)$. When the survey was performed 3 patients (3.3\%) had died from causes unrelated to surgery, and $3(3.3 \%)$ could not be found. Median follow-up for the other 84 patients was 12 years (1-19).

Table IV. Postoperative complications

\begin{tabular}{ll}
\hline Technique & Complication \\
\hline Open procedure (5) & Wound infection (2) \\
& Incisional hernia (2) \\
& Splenic laceration (1) \\
\hline Laparoscopic procedure (4) & Splenic laceration (2) \\
& Pleural opening (1) \\
& Trocar wound bleeding (1) \\
\hline
\end{tabular}

Paraesophageal hernia recurrence, as shown by imaging tests, was found in 4 patients (5\%): in 2 of them a simple open suture had been performed, and the other 2 had undergone laparoscopic Nissen fundoplication. Time between surgery and recurrence was 1,2.2, and 4 years, respectively. Three of them were reoperated, and a fourth reoperation was rejected because of patient comorbidity. Open Nissen fundoplication and mesh placing was performed for the 3 patients.

Furthermore, 3 patients were reoperated because of GER symptoms. Two of them had undergone open D'Or fundoplication; in both cases Nissen's technique was used, in one of them with mesh placement. The third one 
was a laparoscopic Nissen, and the reintervention was a Nissen procedure with mesh placement. Overall reintervention rate was 7\%. An OP was performed in all cases.

Eight patients $(9 \%)$ reported persistent symptoms during follow-up, 4 in the OP group and 4 in the LP group. All of them reported pyrosis and 2 reported dysphagia, symptoms that were controlled using proton-pump inhibitors (PPIs). Eighty patients expressed their satisfaction or high satisfaction with surgery results, including 2 patients who had been reoperated and 2 symptomatic subjects at present.

Even though paraesophageal hernia is not frequent, it may potentially cause very serious complications. There is consensus about repairing once diagnosed, irrespective of whether they are symptomatic or not (1). Nevertheless, some authors still recommend observation for asymptomatic cases taking as a basis a mortality rate associated with surgery that can reach $5.4 \%$, when only $1 \%$ is estimated to need emergency surgery (4).

Surgical repair has shown to be useful, and nowadays the debate focuses on checking whether the laparoscopic approach is as effective and brings the universal advantages of minimally invasive surgery. A great part of authors agree that LP is technically complex and requires extended operative time because of the size and anatomical distortion of paraesophageal hernias. However, OP may be as difficult as LP due to poor access to the upper abdomen and lack of vision (1).

Surgical repair consists of hernia reduction and pillar closure. An antireflux technique is often added since there is evidence of GER symptoms when a fundoplication was not performed, even in those previously asymptomatic (8). There is no consensus about the technique of choice. Some authors argue that esophageal dissection causes GER, while others argue that an anatomic repair would solve this problem, but there are no controlled studies to confirm these hypotheses $(2,9)$. In all patients in our series an antireflux technique or gastropexy was associated, except in 2 patients in which simply a pillar closure was performed. Despite that, 11 patients had symptoms of GER, 3 of whom required reoperation for this reason.

\section{DISCUSSION}

One of the most important complications is hernia recurrence, with an incidence between 9 and $25 \%$ depending on the series $(2,10)$, that may suffer from incarceration and strangulation more often than the primary hernia due to a smaller hiatus after pillar closure. Since recurrence is not always accompanied by clinical manifestations, a close follow-up with imaging tests may be convenient to determine anatomic recurrence without necessarily implying a therapeutic attitude (5).

The use of mesh for repairing seems to reduce the number of relapses, but experience is still limited $(2,11)$.
We have placed mesh in 10 patients and 5 reinterventions with no recurrences to date. We consider that mesh is indicated for large hernias, in which pillar repair may be tense, as well as in relapsing hernia. The literature defines an optimal follow-up time of about 2 years for relapse control (12), but in our case we found one recurrence that showed up after 5 years. Therefore, we think that this monitoring should be extended in order not to underestimate asymptomatic recurrences that may occur at any time during follow-up.

Recent studies argue that the complication rate after LP is lower than after OP (4vs. 16\%) (2). In our series no significant differences in complication rate were reported between both groups (around 10\%), but the abdominal wall complications of OP, absent in the LP group, should be highlighted.

There was no mortality related to surgery in any of both groups.

There was a statistically significant lower hospital stay in the LP group (median 3.5 days) when compared to the OP group (median 9 days), thus confirming what has been described in literature $(1,2)$.

\section{CONCLUSION}

Results after laparoscopic and open surgery are equivalent in terms of efficacy and complication rates, with a significantly shorter hospital stay in the former group. Therefore, we think that laparoscopic surgery should be considered the approach of choice for the treatment of paraesophageal hiatal hernia. The use of mesh has emerged as a promising option to decrease relapse rates. In the absence of conclusive studies its main indication is large hernias and hernia recurrence. Follow-up with imaging tests should be performed for an indeterminate period of time.

\section{REFERENCES}

1. Mehta S, Boddy A, Rhodes M. Review of outcome after laparoscopic paraesophageal hiatal hernia repair. Surg Laparosc Endosc Percu$\tan$ Tech 2006; 16(5): 301-6.

2. Draaisma WA, Gooszen HG, Tournoij E, Broeders IAMJ. Controversies in paraesophageal hernia repair. A review of literature. Surg Endosc 2005; 19: 1300-8.

3. Tsuboi K, Tsukada K, Nakabayashi T, Kato H, Miyazaki T, Masuda $\mathrm{N}$, et al. Paraesophageal hiatus hernia, which has progressed over 8 years: report of a case. Hepatogastroenterology 2002; 49: 992-4.

4. Stylopoulos N, Gazelle GS, Rattner DW. Paraeophageal hernias: operation or observation. Ann Surg 2002; 236: 492-500.

5. Scott Davis S. Current controversies in paraeophageal hernia repair. Surg Clin N Am 2008; 88: 959-78.

6. Schauer PR, Ikramuddin S, McLaughlin RH, Graham TO, Slivka A, Lee KK, et al. Comparison of laparoscopic versus open repair of paraesophageal hernia. Am J Surg 1998; 176: 659-65.

7. Hashemi M, Peters JH, DeMeester TR, Huprich JE, Quek M, Hagen JA, et al. Laparoscopic repair of large type III hiatal hernia: Objective follow-up reveals high recurrence rate. J Am Coll Surg 2000; 190: 553-60.

8. Treacy PJ, Jamieson GG. An approach to the management of parae- 
sophageal hiatus hernias. Aust N Z J Surg 1987; 57: 813-7.

9. Geha AS, Massad MG, Snow NJ, Baue AE. A 32-year experience in 100 patiens with giant paraeophageal hernia: the case for abdominal approach and selective antireflux repair. Surgery 2000; 128: 623-30.

10. Andújar JJ, Papasavas PK, Birdas T, Robke J, Raftopoulos Y, Gagné DJ, et al. Laparoscopic repair of large paraeophageal hernia is associated with a low incidence of recurrence and reoperation. Surg Endosc
2004; 18: 444-7.

11. Targarona EM, Novell J, Vela S, Cerdán G, Bendahan G, Torrubia S, et al. Mid term analysis of safety and quality of life after the laparoscopic repair of paraeophageal hiatal hernia. Surg Endosc 2004; 18: 1045-50.

12. Athanasakis H, Tzortzinis A, Tsiaoussis J, Vassilakis JS, Xynos E. Laparoscopic repair of paraeophageal hernia. Endoscopy 2001; 33: 590-4.

\title{
Hernia de hiato paraesofágica: cirugía laparoscópica vs. cirugía abierta
}

\author{
M. Díez Tabernilla, J. Ruiz-Tovar, R. Grajal Marino, P. Calero García, J. D. Pina Hernández, A. Chames \\ Vaisman, V. Morales Castiñeiras, A. Sanjuanbenito Dehesa, E. Martínez Molina y V. Fresneda Moreno \\ Servicio de Cirugía General y del Aparato Digestivo. Hospital Universitario Ramón y Cajal. Madrid
}

\section{RESUMEN}

Introducción: la hernia hiatal paraesofágica representa el 5$10 \%$ de las hernias hiatales. Su importancia radica en las graves complicaciones que pueden presentar, como el vólvulo gástrico, y se recomienda el tratamiento quirúrgico una vez establecido el diagnóstico.

Material y métodos: estudio retrospectivo de los pacientes intervenidos en nuestro centro de hernia hiatal paraesofágica entre 1985 y 2007.

Resultados: estudiamos 90 casos, 68 mujeres y 22 varones, con edad media de 67,6 años (37-96). Cuarenta y cinco pacientes presentaban pirosis, 34 dolor epigástrico postprandial y 15 disfagia; ocho pacientes fueron diagnosticados como vólvulo gástrico. Se realizaron 81 intervenciones programadas y 9 urgentes. En 47 casos el abordaje fue abierto y en 43 laparoscópico, de los cuales 5 se convirtieron a cirugía abierta. Se realizó funduplicatura D'Or en 35 casos, Nissen en 35, Toupet en 14, cierre simple de pilares en 2 , Narbona en 1 y Lortat-Jakob en 1; en 10 pacientes se colocaron mallas. La tasa de complicaciones en cirugía abierta fue $10,6 \%$ y en laparoscópica $9,5 \%(p>0,05)$. La estancia media fue 9,1 días en cirugía abierta y 3,4 en laparoscópica $(p<0,05)$. En el seguimiento, analizamos 84 pacientes, con una mediana de 12 años (1-19): 15 continuaban sintomáticos, objetivándose recidiva en 8 (5 fueron reintervenidos). El 95,5\% de los pacientes estaban satisfechos con los resultados.

Conclusión: se obtuvieron resultados equivalentes tras cirugía laparoscópica y abierta, con estancia hospitalaria significativamente menor en los primeros. Por ello creemos que se debe considerar la cirugía laparoscópica como abordaje de elección para tratar la hernia hiatal paraesofágica.

Palabras clave: Hernia paraesofágica. Hernia hiatal. Laparoscopia. Reparación herniaria.

\section{INTRODUCCIÓN}

La hernia hiatal paresofágica es una patología poco frecuente que representa entre el 5 y el $10 \%$ de todas las hernias de hiato. Su importancia radica en el riesgo de aparición de complicaciones graves como son la obstrucción o la perforación gástrica o sangrado de su mucosa. Por ello se recomienda el tratamiento quirúrgico en todos los casos (1-3), aunque últimamente algunos autores cuestionan esta actitud, abogando por un manejo conservador expectante en caso seleccionados $(4,5)$. La cirugía abierta (CA) presenta una alta tasa de complicaciones y precisa un tiempo de recuperación prolongado $(1,6)$. En muchas de las recientes series, la cirugía laparoscópica (CL) ha demostrado ser factible y seguro, presentando una menor estancia hospitalaria y menores tasas de complicaciones, aunque algunos autores sugieren una mayor incidencia de recidivas (7).

\section{MATERIAL Y MÉTODOS}

Realizamos un estudio retrospectivo de todos los pacientes intervenidos en nuestro hospital entre los años 1985 y 2007. Mediante revisión de historias clínicas se recogieron los siguientes datos: edad, sexo, manifestaciones clínicas, pruebas diagnósticas utilizadas, técnica quirúrgica empleada, complicaciones postoperatorias y estancia hospitalaria. 
Para evaluar los resultados a largo plazo, realizamos una encuesta de seguimiento entre enero y marzo de 2008 , investigando la presencia de recidivas y el grado de satisfacción de los pacientes. Fueron excluidos los pacientes fallecidos y aquellos de los que no fue posible obtener datos del seguimiento.

El análisis estadístico fue realizado mediante el programa SPSS 12.0 para Windows. Las variables cuantitativas fueron definidas por media (mediana en variables no gaussianas) e intervalo de valores, mientras que en las variables discretas se empleó el número de casos y porcentaje. La comparación de variables discretas se realizó mediante el método de Chi-cuadrado y la diferencia de medias mediante el método de $t$ de Student (Mann-Whitney para variables no normales). Se consideraron estadísticamente significativos los valores $\mathrm{p}<0,05$.

\section{RESULTADOS}

Analizamos un total de 90 pacientes 22 varones $(24,4 \%)$ y 68 mujeres $(75,6 \%)$, con edad media de 67,6 años (37-96). Se indicó tratamiento quirúrgico en todos los casos remitidos a consultas de cirugía general, excepto en aquellos en los que la comorbilidad del paciente contraindicase la intervención quirúrgica. Las manifestaciones clínicas presentadas se resumen en la tabla I. Los síntomas predominantes fueron pirosis $(50 \%)$, dolor epigástrico $(37,8 \%)$, vólvulo gástrico $(8,9 \%)$ y disfagia $(5,6 \%)$. Las pruebas diagnósticas se resumen en la tabla II. En el $83 \%$ de los pacientes se realizó radiografía simple de tórax, siendo de importancia en diagnóstico de sos-

Tabla I. Manifestaciones clínicas

\begin{tabular}{lc}
\hline & Número de casos \\
\hline Pirosis & $45(50 \%)$ \\
Dolor epigástrico & $34(37,8 \%)$ \\
Vólvulo gástrico & $8(8,9 \%)$ \\
Disfagia & $5(5,6 \%)$ \\
Disnea & $5(5,6 \%)$ \\
HDA* & $5(5,6 \%)$ \\
Vómitos & $4(4,4 \%)$ \\
Regurgitación alimentaria & $3(3,3 \%)$ \\
\hline
\end{tabular}

* HDA: hemorragia digestiva alta.

Tabla II. Pruebas diagnósticas

\begin{tabular}{lc}
\hline & Número de casos \\
\hline PEO* & $40(44,4 \%)$ \\
pH-metría & $16(17,8 \%)$ \\
EGD** & $63(70 \%)$ \\
Manometría & $19(21 \%)$ \\
Rx tórax & $75(83,3 \%)$ \\
\hline
\end{tabular}

* PEO: panendoscopia oral; ** EGD: Estudio gastroduodenal. pecha y en los casos de vólvulo gástrico. La paendoscopia oral (PEO) y el estudio gastroduodenal baritado (EGD) fueron las pruebas de mayor rentabilidad diagnóstica, cofirmando el diagnóstico en $91 \%$ de los casos. La pH-metría aportó información sobre la presencia de reflujo gastroesofágico asociado y la manometría aportó información complementaria de la función motora esofágica. Ochenta y un pacientes fueron intervenidos de manera programada (90\%) y 9 de manera urgente (10\%) (8 por vólvulo gástrico y uno por hemorragia digestiva incoercible), estos últimos todos de mediante CA. Las intervenciones realizadas se resumen en la tabla III. Cuarenta y siete pacientes fueron intervenidos mediante CA (52,2\%): las técnicas utilizadas fueron D'Or en 35 casos (74,5\%), Nissen en 8 casos (17\%), cierre de pilares en $2(4,3 \%)$ y Narbona en 1 caso $(2,1 \%)$ y Lortat-Jakob en 1 caso $(2,1 \%) ; 43$ pacientes fueron intervenidos mediante CL $(47,8 \%)$, de los que 5 precisaron reconversión $(11,6 \%)$. Las técnicas utilizadas fueron Nissen en 27 casos $(62,8 \%)$ y Toupet en 16 casos $(37,2 \%)$. Se utilizaron mallas en 8 pacientes en CA $(18,6 \%)$ y en 2 pacientes en CL $(4,3 \%)$. Hubo complicaciones postoperatorias en 5 casos en CA $(10,6 \%)$ y 4 casos en CL $(9,5 \%)(\mathrm{n} / \mathrm{s})$ (Tabla IV). La estancia hospitalaria mediana fue de 9 días en CA y 3 días en CL ( $<<0,05)$. De los 90 pacientes, se obtuvieron datos de $84(93,3 \%)$. En el momento de realización del cuestionario 3 pacientes $(3,3 \%)$ habían fallecido por causas no relacionadas con esta patología, y 3 pacientes $(3,3 \%)$ no fueron localizados. La mediana de seguimiento de estos 84 pacientes fue de 12 años (1-19 años).

Se objetivó recidiva de hernia paraesofágica, documentada con pruebas de imagen en 4 pacientes (5\%), 2 sometidos a cierre simple de pilares por vía abierta y 2 a funduplicatura

Tabla III. Técnica quirúrgica

\begin{tabular}{lcc}
\hline Técnica quirúrgica & $C A$ & $C L$ \\
\hline D'Or & 35 & 0 \\
Nissen & 5 & 30 \\
Toupet & 3 & 13 \\
Cierre simple de pilares & 2 & 0 \\
Narbona & 1 & 0 \\
Lortat-Jakob & 1 & 0 \\
\hline Colocación de malla & 2 & 8 \\
\hline
\end{tabular}

Tabla IV. Complicaciones postoperatorias

\begin{tabular}{ll}
\hline Técnica & Complicación \\
\hline Cirugía abierta (5) & Infección de herida quirúrgica (2) \\
& Eventración (2) \\
& Desgarro esplénico (1) \\
\hline Cirugía laparoscópica (4) & Desgarro esplénico (2) \\
& Apertura pleural (1) \\
& Hemorragia por herida de trocar (1) \\
\hline
\end{tabular}


tipo Nissen laparoscópica. El tiempo transcurrido hasta el diagnóstico de la recidiva fue de 1,2, 2 y 5 años, respectivamente. Tres de ellos fueron reintervenidos y en el cuarto se desestimó la cirugía por patología concomitante. Se realizó colocación de malla y funduplicatura tipo Nissen en los 3.

Además se reintervinieron otros 3 pacientes por presentar síntomas de reflujo gastroesofágico (RGE). En estos pacientes se había realizado funduplicatura tipo D'Or abierta en 2, realizándose en la reintervención funduplicatura tipo Nissen en ambos, con colocación de malla en uno; el tercer paciente reintervenido había sido sometido a una funduplicatura tipo Nissen laparoscópica y la reintervención consistió en colocación de una malla y nueva funduplicatura tipo Nissen.

La tasa global de reintervención fue de $7 \%$. Todas la reintervenciones se realizaron por vía abierta.

Ocho pacientes $(9 \%)$ presentaban síntomas en el momento de la entrevista, 4 intervenidos por CA y 4 por CL: todos ellos presentaban pirosis y 2 disfagia, todo ello controlado con fármacos inhibidores de la bomba de protones.

Ochenta pacientes manifestaron estar satisfechos o muy satisfechos con los resultados de la cirugía, incluyendo 2 pacientes reintervenidos y 2 que presentaban síntomas en la actualidad.

\section{DISCUSIÓN}

Aunque la aparición de la hernia paraesofágica no es frecuente, esta puede presentar complicaciones potencialmente muy graves. Existe consenso en que deben ser reparadas una vez diagnosticadas, independientemente de que sean sintomáticas o no (1). Sin embargo algunos autores aún abogan por la observación de pacientes asintomáticos basándose en una tasa de mortalidad asociada a la cirugía que puede alcanzar hasta 5,4\%, mientras que se estima que sólo el $1 \%$ de los casos precisarán cirugía urgente (4).

La reparación quirúrgica ha demostrado utilidad y el debate actualmente se centra en comprobar si el abordaje laparoscópico es igualmente efectivo y aporta las ventajas universales de la cirugía mínimamente invasiva. Gran parte de los autores coinciden en que la CL es técnicamente compleja, y precisa un tiempo quirúrgico prolongado a causa del tamaño y distorsión anatómica de las hernias paraesofágicas. No obstante la CA puede ser tan dificultosa como la CL por el mal acceso al abdomen superior y la falta de visión (1).

La reparación quirúrgica consiste en reducción de la hernia y cierre de pilares. A esto se suele añadir una técnica antirreflujo, ya que hay evidencia de síntomas de RGE postoperatorio en pacientes en los que no se realizó funduplicatura incluso en pacientes previamente asintomáticos (8). No existe consenso acerca de la técnica de elección. Algunos autores defienden que la disección esofágica induce RGE, mientras que otros mantienen que la reparación anatómica solucionaría este problema, pero no existen estudios controlados que confirmen estas hipótesis $(2,9)$. En todos los pacientes de nuestra serie se asoció una técnica antirreflujo o pexia gástrica, exceptuando 2 pacientes, en los que simplemente se cerraron los pilares. A pesar de ello 11 pacientes presentaron síntomas de RGE, de los cuales 3 precisaron reintervención por este motivo.

Una de las complicaciones más importante es la recidiva de la hernia, con una incidencia que oscila entre 9 y $25 \%$ según series $(2,10)$, que se asocia a incarceración y estrangulación en mayor medida que la primaria, por menor tamaño del hiato después de la reparación de los pilares. Dado que la recidiva, no siempre se acompaña de presencia de manifestaciones clínicas, puede ser conveniente realizar un seguimiento estrecho con pruebas de imagen que determinen la recidiva anatómica, sin que ello implique necesariamente una actitud terapéutica (5).

El uso de mallas para la reparación parece disminuir el número de recidivas, pero la experiencia es aún escasa $(2,11)$. En nuestros pacientes hemos colocado malla en 10 pacientes, y en 5 reintervenciones, sin recidiva hasta el momento actual. Pensamos que la malla está indicada en hernias de gran tamaño, en las que la reparación de los pilares puede resultar a tensión, y en las hernias recidivadas. La literatura define un tiempo de seguimiento óptimo de unos 2 años para el control de recidiva (12), pero en nuestra serie hemos encontrado un caso que recidivó a los 5 años. Por ello pensamos que este seguimiento debe prolongarse para no infravalorar recidivas asintomáticas que pueden aparecer en cualquier momento del seguimiento.

Estudios recientes defienden que la tasa de complicaciones tras CL es menor que tras CA (4 frente a 16\%) (2). En nuestra serie no se observó una diferencia significativa de tasa de complicaciones entre los 2 grupos, en ambos en torno al $10 \%$, destacando en el grupo de CA las complicaciones de la pared abdominal, ausentes en el grupo CL.

No hubo mortalidad en ambos grupos.

Se observó una menor estancia hospitalaria estadísticamente significativa en el grupo CL (mediana 3,5 días) frente al grupo CA (mediana 9 días), confirmando lo descrito en la literatura $(1,2)$.

\section{CONCLUSIÓN}

Los resultados obtenidos tras cirugía laparoscópica y abierta son equivalentes en cuanto a eficacia y tasa de complicaciones, con una estancia hospitalaria significativamente menor en los primeros. Por ello creemos que se debe considerar la cirugía laparóscopica como abordaje de elección para el tratamiento de la hernia de hiato paraesofágica. El uso de mallas se perfila como prometedor para disminuir la tasa de recidivas. En ausencia de estudios concluyentes su principal indicación sería hernias de gran tamaño y hernias recidivadas. El seguimiento con pruebas de imagen debe realizarse durante un tiempo indeterminado. 